\title{
Simulation of atmospheric drag effect on low Earth orbit satellites during intervals of perturbed and quiet geomagnetic conditions in the magnetosphere- ionosphere system
}

\author{
Victor Uchenna. J. Nwankwo \\ Space-APRAWP Laboratory, \\ Department. of Physics \\ Anchor University \\ Lagos, Nigeria \\ vnwankwo@aul.edu.ng \\ Wahabbi Akanni \\ Department of Mathematical Sciences, \\ Anchor University, \\ Lagos, Nigeria \\ wakanni@aul.edu.ng \\ Jean-Pierre Raulin \\ Center for Radio Astronomy and \\ Astrophysics Mackenzie, \\ Presbyterian University Mackenzie, \\ Sao Paulo, Brazil \\ raulin@craam.mackenzie.br
}

\author{
William Denig \\ St. Joseph College of Maine, \\ Standish, ME 04084, USA \\ wdenig@sjcme.edu.net \\ Johnson Fatokun \\ Department of Mathematical Sciences, \\ Anchor University, \\ Lagos, Nigeria \\ jfatokun@aul.edu.ng \\ Emilia Correia \\ Center for Radio Astronomy and \\ Astrophysics Mackenzie \\ Presbyterian University Mackenzie, \\ Sao Paulo, Brazil \\ ecorreia@craam.mackenzie.br
}

\author{
Muyiwa P. Ajakaiye \\ Space-APRAWP Laboratory, \\ Department. of Physics \\ Anchor University \\ Lagos, Nigeria \\ majakaiye@aul.edu.ng \\ Sandip K. Chakrabarti \\ Indian Centre for Space Physics, \\ Kolkata 700084, India \\ sandipchakrabarti9@gmail.com
}

John E. Enoh

Interorbital systems,

Mojave, CA 93502-0662

enojoneno@gmail.com
Abstract-In this work, we simulate the effect of atmospheric drag on two model low Earth orbit (LEO) satellites with different ballistic coefficient during 1-month intervals of geomagnetically disturbed and relatively quiet conditions, to understand how solar and geomagnetic activity modulates satellites trajectory in Earth's orbit. Our results showed that geomagnetic disturbances on the upper atmosphere associated with high solar activity caused a total decay of $2.77 \mathrm{~km}$ and $3.09 \mathrm{~km}$ for SAT-A and Sat-B, respectively during the 1-month period, but only about $0.52 \mathrm{~km}$ and $0.65 \mathrm{~km}$, respectively during the interval of relatively quiet geomagnetic condition. The mean orbit decay rates (ODR) of the two satellites are $\sim 90 \mathrm{~m} / \mathrm{day}$ and $\sim 100 \mathrm{~m} / \mathrm{day}$, respectively during the perturbed regime, while the respective values for the relatively quiet regime are $\sim 17 \mathrm{~m} / \mathrm{day}$ and $21 \mathrm{~m} / \mathrm{day}$. Within the two regimes, further analysis and simulation of the satellites' responses during 12-day intervals of elevated solar and geomagnetic activity and exceptionally quiet activity showed that SAT-A and Sat-B decayed by about $1.13 \mathrm{~km}$ and $1.27 \mathrm{~km}$, respectively during the former regime, while the respective decay for the latter regime are $0.16 \mathrm{~km}$ and $0.20 \mathrm{~km}$. The respective mean ODR are $101.38 \mathrm{~m} / \mathrm{day}$ and $113.22 \mathrm{~m} / \mathrm{day}$ (for elevated activity), and $14.72 \mathrm{~m} /$ day and $18.52 \mathrm{~m} / \mathrm{day}$ (exceptionally quiet). Sat-B has larger values of height decay (h) and ODR in both regimes, and therefore affected by atmospheric drag force more than Sat-A, because its ballistic coefficient is higher. The results of our simulation confirm (i) the dependence of atmospheric drag force on satellites ballistic coefficient, and (ii) geomagnetic storms being the leading driver of large-scale disturbances in the coupled magnetosphere-ionosphere-thermosphere systems, and consequently the leading 'perturber' of satellites' motion in low Earth orbit. Our model can be useful for situational awareness and mitigation of the potential threat posed by solar activity in modulating satellites trajectories.

Atmospheric drag, ballistic coefficient,LEO satellites, solargeomagnetic activity

\section{INTRODUCTION}

Atmospheric drag describes the force exerted on any object moving through the atmospheric medium, having orientation in the direction of relative motion with the tendency of slowing the motion of the body. Spacecrafts moving through atmospheric medium experience atmospheric drag force, which constantly takes energy away from the orbit [1-3]. Atmospheric drag is the strongest force affecting the motion of satellites in low Earth orbit (LEO) especially at altitudes $<800 \mathrm{~km}$ [4]. Solar activity enhances atmospheric drag on Low Earth orbiting satellites (LEOSs) and their effects on the space systems can be profound depending on the severity of solar activity [3]. Atmospheric drag can cause satellites premature re-entry, as well as difficulty in maneuvering, identifying and tracking of satellites and other space objects, and prediction of their lifetime and actual re-entry ([4], and references therein). Premature re-entry of spacecrafts could be due to draginduced accelerated orbital decay. Under this scenario a satellite gradually decays from orbit (loosing height) and reenters the Earth atmosphere if appropriate measure or orbit maintenance is not done in timely manner (see, Fig. 1). Examples of spacecrafts that re-entered the atmosphere in the past include Skylab (launched 14 May, 1973, re-entered 11 July 1979), Russian RORSATs, Kosmos-954 (launched 18 September 1977, re-entered 24 January, 1978) and Kosmos-1402 (launched 30 August, 1982, re-entered 7 February, 1983) etc. [3].

The extent to which LEOSs are affected by atmospheric drag also depends on their injected height $(h)$ and ballistic coefficients $(B)$. Satellite's ballistic coefficient is given by $B=C_{d} A_{s} / m_{s}$, where $C_{d}$ is the drag coefficient, $A_{s}$ is the projected area and $m_{s}$ is the mass of the satellite. Satellites having smaller values of $B$ experiences less drag force, and 
tend to stay longer in the orbit than those with larger value $[2,3]$.

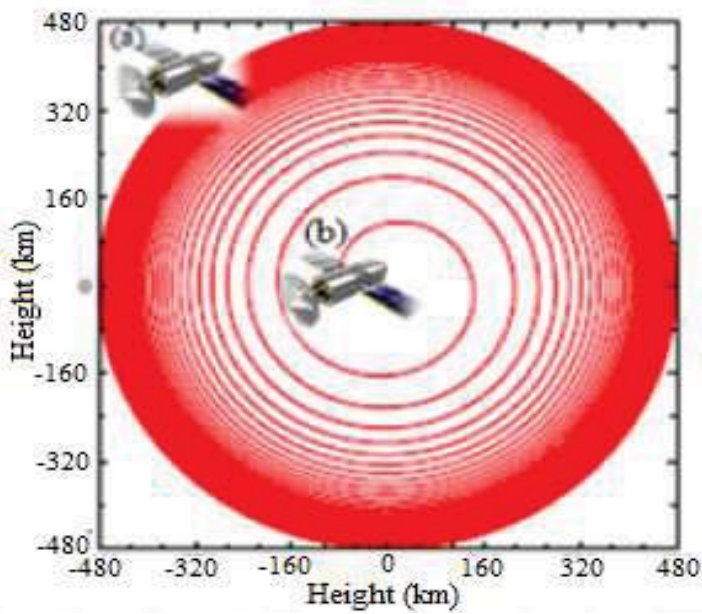

Fig. 1.Orbital decay scenario of low Earth orbiting Satellite (LEOS) due to atmospheric drag. A LEOS in orbit can drop from a point a tob under the influence of atmospheric drag (adapted from [3]).

Solar activity describes all forms of output from the Sun including light, solar wind, and energetic events and particles. Streams of both high and low speed energised charged particles or plasma (in solar wind) with embedded magnetic field are constantly flowing out of the Sun. When energetic particles from the solar wind collide and interact with the Earth's magnetic fields, it enhances geomagnetic activity, which can have important implication on the magnetosphere-ionosphere system. High speed solar wind streams (HSS) can interact with preceding low speed streams (LSS), forming a dense corotating interaction regions (CIRs) that can potentially increase geomagnetic activity. Sometimes, the Sun also releases large-scale, high-mass, eruptions of plasma in a phenomenon known as coronal mass ejections (CMEs), which propagates into the interplanetary space. When directed towards Earth, CIRs and CMEs can potentially cause geomagnetic storms (the leading driver of large-scale coupled magnetosphere-ionosphere perturbations in the geospace).

One other important solar activity with lesser geomagnetic implications, is the solar flares. Solar flare is a strong, momentary release of great amount of magnetic energy (from the Sun) with the potential of heating up materials to many millions of degrees. Electromagnetic (EM) radiation and energetic particles are also released during solar flares, with the dominant emission being the X-ray and Extreme Ultra Violet (EUV) that can cause abrupt increase in atmospheric ionisation rate (known as sudden ionospheric disturbance (SID)).

Observations of the sunspot number (and other solar indices) over two centuries, have shown that solar activity exhibit approximately 11-year cycle during which the sunspot count move from minimum to maximum values. To demonstrate the solar cycle, we show in Fig. 2, the variations in archived (1992-2012) and predicted (2013-2020) sunspot number, solar radio flux (F10.7) and geomagnetic $A_{p}$ index for 28 years. Solar flares and CMEs and associated phenomena (e.g., geomagnetic storms) occur more frequent during the solar maximum but significantly reduced during the solar minimum. Consequently, solar and geomagnetic indices usually reach their 'high' and/or marked by large fluctuation around the solar maximum. The high energy charged particles and EM radiation from solar and geomagnetic activity form important channel through which the Earth's atmosphere is impacted by the Sun. These energetic charged particles and EM radiation heats up and expands the upper atmosphere (or ionosphere) leading to significant increase in atmospheric (or thermospheric) density. Consequently, atmospheric drag on satellites increases, perturbing the motion of LEO spacecrafts, and causing accelerated orbit decay (as depicted in Fig. 1).

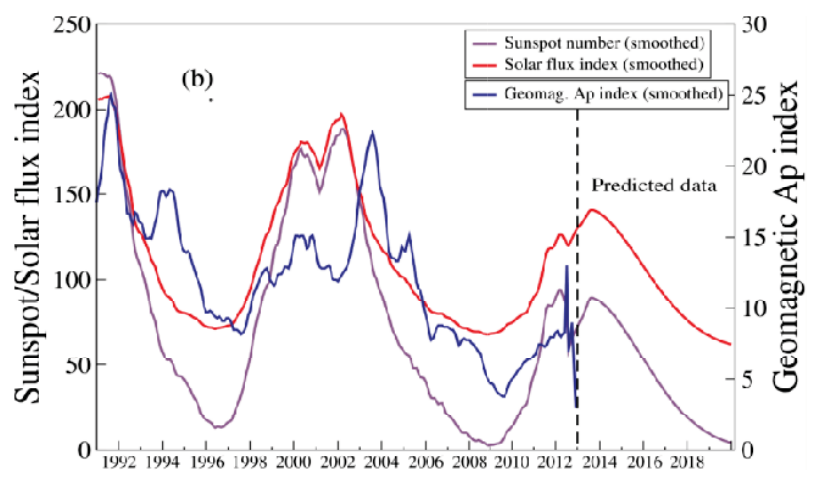

Fig. 2. Variation in archived (1992-2012) and predicted (2013-2020) sunspot number, solar radio flux (F10.7) and geomagnetic $A_{p}$ index for 28 years [5].

\section{IMPORTANT APPLICATION OF THE STUDY}

Atmospheric drag perturbs the motion of LEOSs, and increases the risk of satellites collision due to the increased margins of error in spacecraft positioning. On February 10, 2009, a U.S. communication satellite (Iridium 33) collided with a Russian satellite in orbit (Cosmos 2251) at an altitude of about $800 \mathrm{~km}$ [6]. Cosmos 2251 was a defunct satellite (space debris) in orbit, while Iridium 33 was a normal operational satellite providing telecommunication services when the accident occurred. The study of atmospheric dragassociated risk is important because of the increasing number of both active space missions and debris objects that is posing serious challenge to the society in the area of space traffic management. Also, the issues relating to planned possible launch of new facilities (e.g., UK-licensed megaconstellations etc.) makes this subject increasingly important to satellite operators and stakeholders [7]. Although the incident of Iridium-Cosmos collision is cited as an example of risk of possible collision due to drag-induced error in spacecraft positioning, we do not explicitly attribute this unprecedented accident to drag force impact. Authors recommend that readers consult related articles for more detail on the incident (e.g., [6], and references therein), and the extended version of this paper. Space agencies unequivocally acknowledge the potential threat posed by solar activity in modulating satellites trajectory, and are making appreciable effort towards addressing the issue. As an example, the European Space Agency (ESA) is currently addressing space weather associated risks within the framework of the Space Situational Awareness (SSA) programme. One important mitigation approach (among others) to solar activity impact on satellites trajectory is the development and implementation of appropriate model that can mimic perturbed scenarios on LEOSs that are expected under hash space environmental conditions [8]. Therefore, this work is of practical importance as the resulting model and/or simulation could provide resources for forecast, situational awareness and impact mitigation. 


\section{METHOD AND SCOPE OF THE STUDY}

In this work we model LEO satellites trajectory during disturbed and relatively quiet geomagnetic conditions, to understand how solar and geomagnetic activity influence atmospheric drag and satellite orbit. The model will be implemented on two satellites with different ballistic coefficients (given in Table 1). To start with, we analyse parameters that describe variations in solar and geomagnetic activity including solar wind speed $\left(\mathrm{V}_{\mathrm{sw}}\right)$ and associated particle density (PD), disturbance storm time (Dst), interplanetary magnetic field (IMF) $\mathrm{B}_{\mathrm{y}}$ and $\mathrm{B}_{\mathrm{z}}$, and auroralelectrojet (AE) indices from the OMNI solar wind 1 AU

data upstream(https://omniweb.gsfc.nasa.gov/form/omni min.htm 1). The velocity, density, intensity and direction of the solar wind plasma and associated magnetic field controls the processes by which energy is transferred from the solar wind to the magnetosphere-ionosphere-thermosphere (MIT) system and also influences the structure of the surrounding Earth's magnetic field ([3], and references therein). The Dst and $\mathrm{AE}$ indices describe the degree of geomagnetic disturbance. The southward $\mathrm{B}_{\mathrm{z}}$ component causes geomagnetic storms and significantly contributes to energy transfer from the solar wind sector to the MIT system [9]. The regimes for which analysis were made are (i) 1-31 July,2000, representing interval of geomagnetically perturbed condition and (ii) 1-31 July ,2006, representing the interval of relatively quiet geomagnetic activity.

\section{Perturbed Geomagnetic Condition DURING 1-31 JULY, 2000}

The year 2000 witnessed a significant increase in solar activity, and later reacheda peak (solar maximum) in 2001 being the 23rd solar cycle. Fig. 3 shows 1-hour averaged variations in solar wind speed $\left(\mathrm{V}_{\mathrm{sw}}\right)$, proton density $(\mathrm{PD})$, disturbance storm time (Dst), IMF $\mathrm{B}_{\mathrm{y}}$ and $\mathrm{B}_{\mathrm{z}}$ components and auroralelectrojet (AE) index during 1-31 July, 2000.Solar and geomagnetic activity was significantly high during the interval, leading to large variations or fluctuations in the indices.Data showed that the average F10.7 and $A_{p}$ index during the interval (1-31 July, 2000) was approximately 200 and 22, respectively.The F10.7 index, indirectly estimates upper atmospheric heating from solar energetic particles and solar EUV, while $A_{p}\left(\right.$ or $\left.K_{p}\right)$ index estimates additional Joule heating associated with geomagnetic activity (Nwankwo et al., 2015 and references therein). Particularly important, is the condition in the solar wind during the one month period - significant fluctuations (and increase) in the PD during 1-4, 9-15 and 25-29. The corresponding fluctuation and subsequent increase in $\mathrm{V}_{\mathrm{sw}}$ during 9-15 have important consequence on the MIT system.

The ultimate manifestation of this solar wind forcing is the occurrence of strong geomagnetic storms with Dst of about $-300 \mathrm{nT}$ (see, Fig. 3c(left panel)) on 15 July. A geomagnetic storm occurs, $\mathrm{B}_{\mathrm{z}}$ turns southward (see, Fig.3e(left panel)), and the value of Dst drops to $\geq-50$ nT.The condition of the magnetic field components $\left(\mathrm{B}_{\mathrm{y}}, \mathrm{B}_{\mathrm{z}}\right)$ suggests significant energy transfer to MIT system (from the solar wind), and the increase in AE corresponding with the storm peak indicates enhanced Ionospheric currents activity (in the auroral zone) and a strong coupling between the IMF and the earth's MIT system. Clearly, the upper atmosphere was significantly disturbed during the interval due to the geomagnetic storms.

\section{GEOMAGNETIC QUIET CONDITION DURING 1- 31 JULY, 2006}

Solar activity was at the decline in 2006 but the minimum activity occurred in 2008-2009 (see, Fig. 2.). The declining or solar minimum phase is usually marked by drastic reduction in solar energetic events. Fig. 3 also shows 1-hour averaged variations in $V_{s w}, P D$, Dst, IMF $B_{y}$ and $B_{z}$ components and AE indices during 1-31 July, 2006. When compared with July 2000 scenario we see a relatively less variation or fluctuation in the parameters. However, there are intervals marked with significant fluctuations. These intervals demonstrate the occurrence of isolated but sporadic and/or spontaneous spikes in solar activity, as well as other important energetic phenomena during the declining phase of solar activity. One example of such phenomena is the occurrence of CIRs in solar wind (explained in section 2). CIR occurs when HSS interacts with preceding LSS, forming an interface of high density plasma region. This corotating interaction region of high density plasma can interact with the Earth's magnetosphere and produces geomagnetic disturbances and/or storms. The conditions in $\mathrm{V}_{\mathrm{sw}}$ and PD during 3-5, 9-12 and 26-27 July 2006 and the resulting fluctuations in the rest of the parameters (Dst, $\mathrm{B}_{\mathrm{y}}, \mathrm{B}_{\mathrm{z}}, \mathrm{AE}$ ) are more like scenarios of CIR-induced disturbances. The energy transferred into the MIT system by the solar wind forcing at the time appears not to be strong enough to cause geomagnetic storms. Nonetheless, the MIT system was geomagnetically disturbed during the intervals. We note that solar and geomagnetic activity was relatively quiet during 921 July 2006. Our simulation will highlight this interval of relative geomagnetic quiet and compare the results with the moments of severe geomagnetic disturbances due to the strong storm around 14-15 July, 2000.

\section{MODELING ATMOSPHERIC DRAG EFFECT ON LEO SATELLITES' TRAJECTORY}

We formulated and solved a set of coupled differential equations in previous work, to obtain instantaneous position, velocity components and acceleration of typical LEO satellites under the influence of atmospheric drag force in orbit (e.g., [4,10-12]). We used the spherical polar coordinate system $(r, \theta, \varnothing)$ with origin at the center of the Earth, and assumed that the satellite always remains in the same plane (i.e., $\theta=$ constant) while changing its position through the azimuthal parameters $(\varnothing, \varnothing)$. We compute the orbital decay due to atmospheric drag on the satellite from the following set of equations.

$$
\begin{aligned}
& v_{r}=-\emptyset r^{2}\left(\frac{A_{s} C_{d}}{m_{s}}\right) \\
& \dot{r}=v_{r} \\
& \ddot{\emptyset}=-\frac{1}{2} r \rho \dot{\emptyset}^{2}\left(\frac{A_{s} C_{s}}{m_{s}}\right) \\
& \dot{\emptyset}=\frac{v_{\phi}}{r}
\end{aligned}
$$

$\mathrm{V}_{\mathrm{r}}$ and $\mathrm{v}_{\varnothing}$ are the radial and tangential velocity components, respectively, $r$ is the instantaneous radius of the orbit, $\rho$ is atmospheric density. The parameters $\mathrm{C}_{\mathrm{d}}, \mathrm{A}_{\mathrm{s}}$ and $\mathrm{m}_{\mathrm{s}}$ have been described in section 1 . The ballistic coefficient of the model LEOSs is given in Table 1 below. 

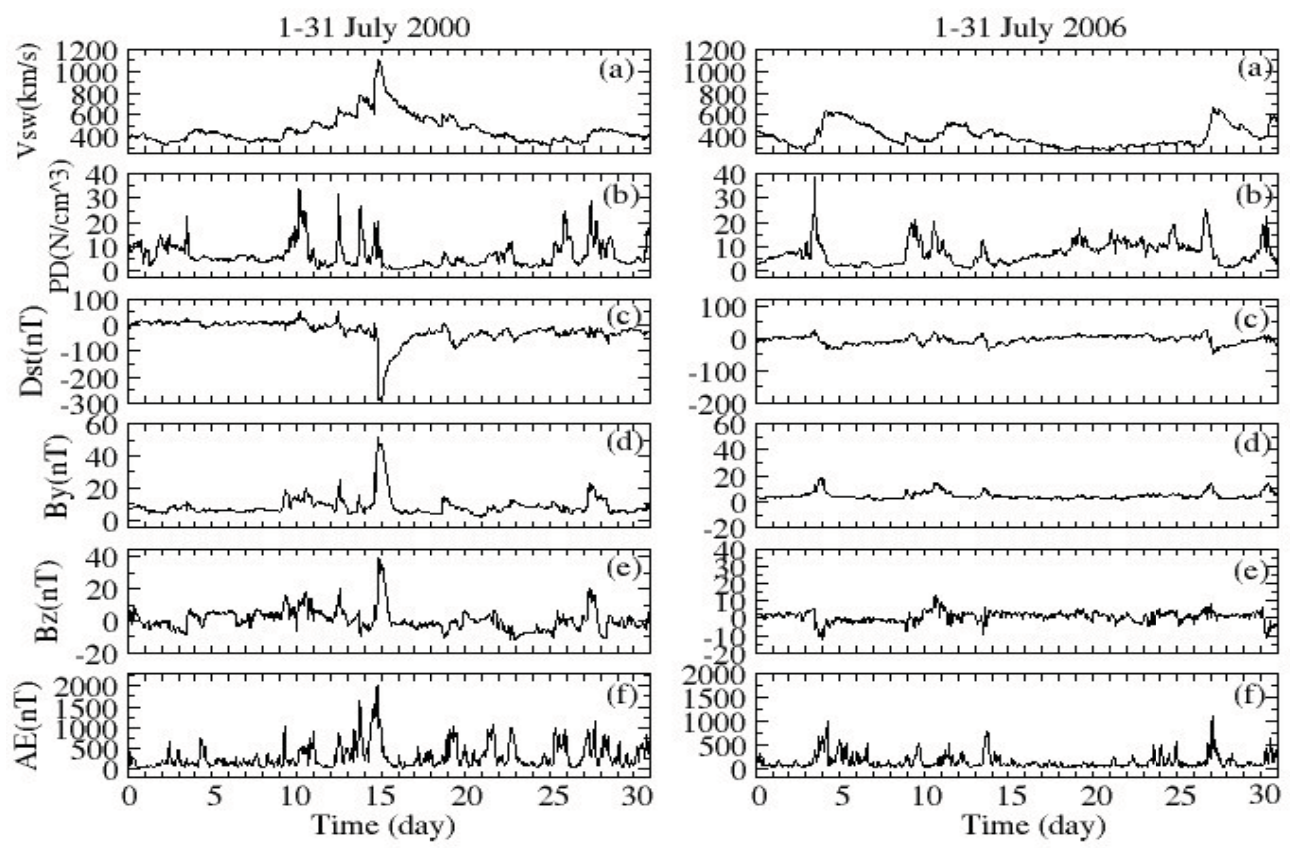

Fig. 3. 1-hour averaged variations in solar wind speed $\left(\mathrm{V}_{\mathrm{sw}}\right)$, proton density (PD), disturbance storm time (Dst), IMF $\mathrm{B}_{\mathrm{y}}$ and $\mathrm{B}_{\mathrm{z}}$ components and auroralelectrojet (AE) index during (i) 1-31 July, 2000 and (ii) 1-31 July, 2006.
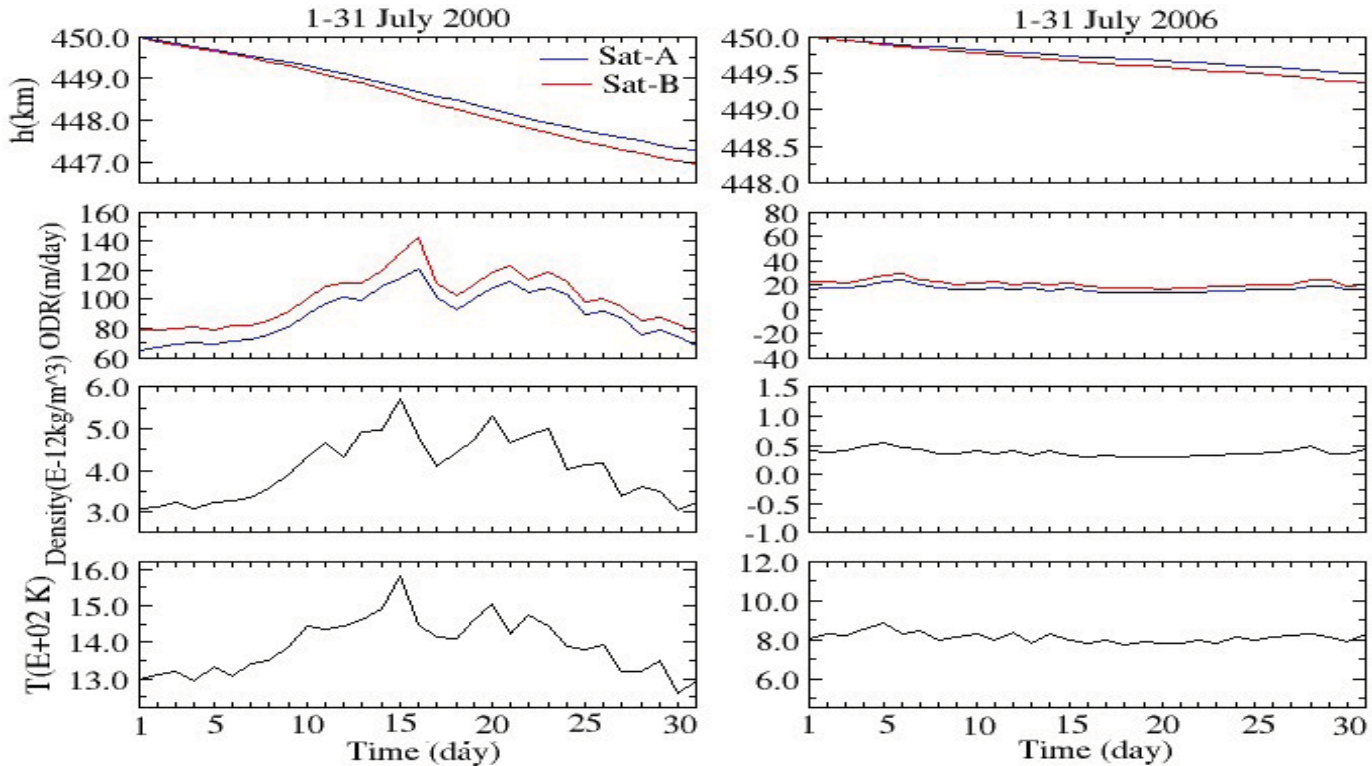

Fig. 4. Mean daily variations altitude (h), orbit decay rate (ODR), thermospheric temperature (T) and density ( $\rho$ ) for Sat-A and Sat-B during (a) 1-31 July, 2000 and (b) 1-31 July, 2006.

TABLE I.SATELLITES ORBITAL AND BALLISTIC PARAMETERS USED IN THE STUDY

\begin{tabular}{|c|c|c|c|c|c|}
\hline & $\mathrm{h}(\mathrm{km})$ & $\mathrm{m}(\mathrm{kg})$ & $\mathrm{A}_{\mathrm{s}}\left(\mathrm{m}^{2}\right)$ & $\mathrm{C}_{\mathrm{d}}$ & $\mathrm{B}$ \\
\hline Sat A & 450 & 250 & 0.25 & 2.2 & 0.002200 \\
\hline Sat B & 450 & 522 & 0.72 & 2.2 & 0.003034 \\
\hline
\end{tabular}

\section{ATMOSPHERIC DENSITY PROFILE}

The effects of atmospheric drag on LEOSs and hence, the rate at which satellites decays from orbital largely depends on the atmospheric density, which is heavily influence by solar and geomagnetic activities. Therefore, accurate computation of atmospheric drag effect, and prediction of a satellite's lifetime or re-entry requires good estimation of atmospheric density profile. The upper atmosphere (or thermosphere) exhibits large solar cycle changes in temperature, density, composition and winds in the amount of absorbed solar radiation [13]. Many atmospheric density models have been developed over the years with good approximation, and are frequently being upgraded as new data or insight becomes available (e.g., the Naval Research Laboratory Mass Spectrometry and Incoherent Scatter Extended 2000 (NRLMSISE-00), the drag temperature model 2000 (DTM-2000), Jacchai-Bowman 2008 (JB2008)). The atmospheric/thermospheric temperature $(\mathrm{T})$ and density ( $\rho)$ profile used in this work were obtained using the NRLMSISE-00 model. The NRLMSISE-00 empirical atmospheric model is composed of parametric and analytic 
approximations to physical theory for vertical structure of the atmosphere as a function of time, location, solar and geomagnetic activity (via $\mathrm{A}_{\mathrm{p}}$ and F10.7) (see, [14]).

\section{RESULTS AND DISCUSSION}

\section{A. Atmospheric drag effect on LEOSs during perturbed geomagnetic condition}

The result of our simulation arising from the solution of the above coupled differential equation is presented here. Fig. 4 shows the mean daily variations in the altitude (h), orbit decay rate (ODR), thermospheric temperature (T) and density $(\rho)$ for Sat-A and Sat-B during (i) 1-31 July, 2000 and 1-31 July, 2006. Sat-A decayed by about $2.77 \mathrm{~km}$ during the entire month, while Sat-B decayed by about $3.09 \mathrm{~km}$. This implies that the effect of atmospheric drag on Sat B was more than that experienced by Sat-A, because its ballistic coefficient is higher. The range of ODR for Sat-A and Sat-B for the interval are $65-120 \mathrm{~m} /$ day and $78-142 \mathrm{~m} /$ day, respectively. As expected, the values of ODR are higher for Sat-B. The thermospheric temperature varied between 1156 $\mathrm{K}$ and $1580 \mathrm{~K}$, while the density varied between $2.4 \times 10^{-12}$ $\mathrm{kg} / \mathrm{m}^{3}$ and $5.7 \times 10^{-12} \mathrm{~kg} / \mathrm{m}^{3}$. Data showed that the severe geomagnetic storm lasted for about 3 days; effectively commenced at about 20:00 (UT) on 15 July, reached a peak around 00:00 (UT) on 16 July, and then recovered until 18:00 (UT) on 17 July. There were also recurrent but mild storms between 20 and 24, and on 29 July (see, Fig. 3c(left panel)).

Interestingly, the result of our simulation showed a corresponding increase in $\mathrm{T}$ and $\rho$, and consequently in ODR throughout the duration of the main storm (with the largest values occurring around the major storm day on 15-16 July), and on the days of the recurrent storms (see, Fig. 4(left panel)). The mean thermospheric $T$ and $\rho$ on the storm's peak day (16 July) are $1580 \mathrm{~K}$ and $5.7 \times 10^{-12} \mathrm{~kg} / \mathrm{m}^{3}$, and the corresponding values of ODR for Sat-A and Sat-B are 123 $\mathrm{m} /$ day and $142 \mathrm{~m} /$ day, respectively. It is therefore clear that MIT system was heavily disturbed by the geomagnetic storms, with strong consequences on the trajectory of the satellites in low Earth orbit. This result confirms the dependence of atmospheric drag force on satellites ballistic coefficient, and geomagnetic storms being the leading driver of large-scale disturbances in the coupledMIT dynamics in the geospace environment.

\section{B. Atmospheric drag effect on LEOSs during low geomagnetic activity}

In Fig 4 (right panel) we also show the mean daily variations in $h$, ODR, thermospheric T and $\rho$ for Sat-A and Sat-B during 1-31 July 2006. During this 1-month period of relatively low geomagnetic activity, T varied between $770 \mathrm{~K}$ and $880 \mathrm{~K}$, while $\rho$ varied between $0.33 \times 10^{-12}$ and $0.55 \times 10^{-}$ ${ }^{12} \mathrm{~kg} / \mathrm{m}^{3}$. Sat-A and Sat B decayed by only about $0.52 \mathrm{~km}$ and $0.65 \mathrm{~km}$, respectively. The range of ODR for the two model satellites are $13-23 \mathrm{~m} /$ day and $16-29 \mathrm{~m} /$ day, respectively. There was no geomagnetic storms recorded during the entire month but we note two important spikes in solar activity around 6 and 27 July (not strong enough to produce a storm) that resulted in respective ODR of $23 \mathrm{~m} /$ day and $19 \mathrm{~m} /$ day for Sat-A, and $29 \mathrm{~m} /$ day and $23 \mathrm{~m} /$ day for Sat-B. Nonetheless, variations in $\mathrm{T}$ and $\rho$, and the satellites' $\mathrm{h}$ and ODR were comparatively very small when juxtaposed with the scenario in July 2000.

\section{Intervals of elevated and exceptionally quiet geomagnetic activity scenarios compared}

We further highlight the interval of elevated solar and geomagnetic activity during 1-31 July,2000, and the interval of exceptionally quiet activity during 1-31 July, 2006. The selected intervals for the two regimes are 9-20 July, 2000 and 15-26 July, 2006 (12 days each). We compare variations in solar and geomagnetic indices during these intervals with the effect produced on the satellites' trajectory parameters ( $h$, ODR) due to the corresponding solar-induced perturbations in $T$ and $\rho$ profiles. Fig. 5 shows 1-hour averaged variations in $\mathrm{V}_{\mathrm{sw}}, \mathrm{PD}$, Dst, IMF $\mathrm{B}_{\mathrm{y}}$ and $\mathrm{B}_{\mathrm{z}}$ components and $\mathrm{AE}$ indices during (i) 9-20 July, 2000 and (ii) 15-26 July, 2006. The former interval represents the interval of elevated solar and geomagnetic activity, while the later represent interval of exceptionally quiet solar and geomagnetic activity. In Fig. 6 we show the corresponding mean daily variations in h, ODR, thermospheric T and $\rho$ for Sat-A and Sat-B for the selected intervals. During the elevated solar activity Sat-A decayed from orbit by a total of about $1.14 \mathrm{~km}$ and the ODR increased from $81.46 \mathrm{~m} /$ day on 9 July to $120 \mathrm{~m} /$ day at the peak of the major storm on 16 July. Similarly, Sat-B decayed by about $1.27 \mathrm{~km}$, and the ODR increased from $91.85 \mathrm{~m} /$ day to $142 \mathrm{~m} /$ day (see, fig 6(a-b)-left panel). On the other hand, Sat-A and Sat-B decayed by a total of only $0.16 \mathrm{~km}$ and 0.20 $\mathrm{km}$, respectively during the interval of exceptionally 'quiet' geomagnetic activity (see, Fig. 6(a-b)-right panel). The ODR of both satellites dropped from respective values of 17.17 $\mathrm{m} /$ day and 21.25 on 15 July 2006 to a low value of 13.41 $\mathrm{m} /$ day and $16.67 \mathrm{~m} /$ day as solar activity decreased further. The stark contrast between the two regimes is that solar activity can impact up to 6 times as much (or more) on the satellites trajectory during interval of elevated geomagnetic disturbances. However, the extent of impact depends on the intensity and duration of driving solar energetic event and the ballistic coefficient of the satellite.

\section{CONCLUSION}

Energetic charged particles and EM radiation from solar energetic event form important channel through which the Earth's atmosphere is impacted by the activity of the Sun. They heat up and expand the upper atmosphere leading to significant increase in atmospheric temperature and density. This condition increases atmospheric drag on satellites, which perturbs their motion and causes accelerated orbit decay. In this work, we simulated the effect of atmospheric drag on two model LEO satellites with different ballistic coefficient during 1-month intervals of geomagnetically disturbed and relatively quiet conditions to understand how solar and geomagnetic activity influence atmospheric drag and satellite orbit. Our results showed that geomagnetic disturbances on the upper atmosphere associated with high solar activity caused a total decay of $2.77 \mathrm{~km}$ and $3.09 \mathrm{~km}$ for SAT-A and Sat-B, respectively during the 1-month period (1-31 July 2000), but only about $0.52 \mathrm{~km}$ and 0.65 $\mathrm{km}$, respectively during the interval of relatively quiet geomagnetic condition (1-31 July 2006). The mean orbit decay rates (ODR) of the two satellites are $\sim 90 \mathrm{~m} /$ day and $\sim 100 \mathrm{~m} /$ day, respectively during the perturbed regime, while the respective values for the relatively quiet regime are $\sim 17$ $\mathrm{m} /$ day and $21 \mathrm{~m} /$ day. Within the two regimes, further analysis and simulation of the satellites' responses during 12-day intervals of elevated solar and geomagnetic activity and exceptionally quiet activity showed that SAT-A and Sat-B decayed by about $1.13 \mathrm{~km}$ and $1.27 \mathrm{~km}$, respectively during 

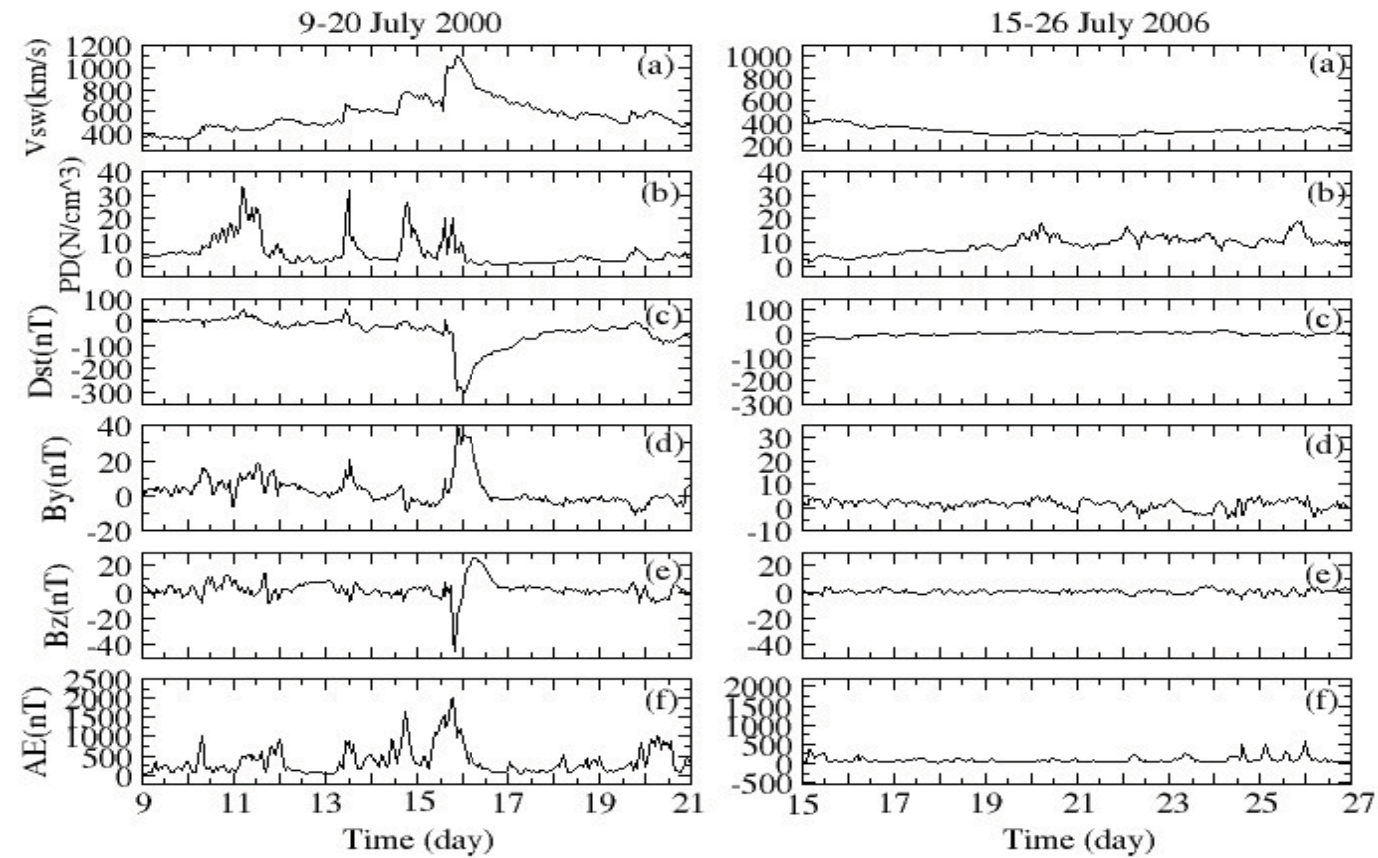

Fig. 5. 1-hour averaged variations in $\mathrm{V}_{\mathrm{sw}}$, PD, Dst, IMF $\mathrm{B}_{\mathrm{y}}$ and $\mathrm{B}_{\mathrm{z}}$ components and AE index during (i) 9-20 July 2000 and (ii) 15-26 July 2006.
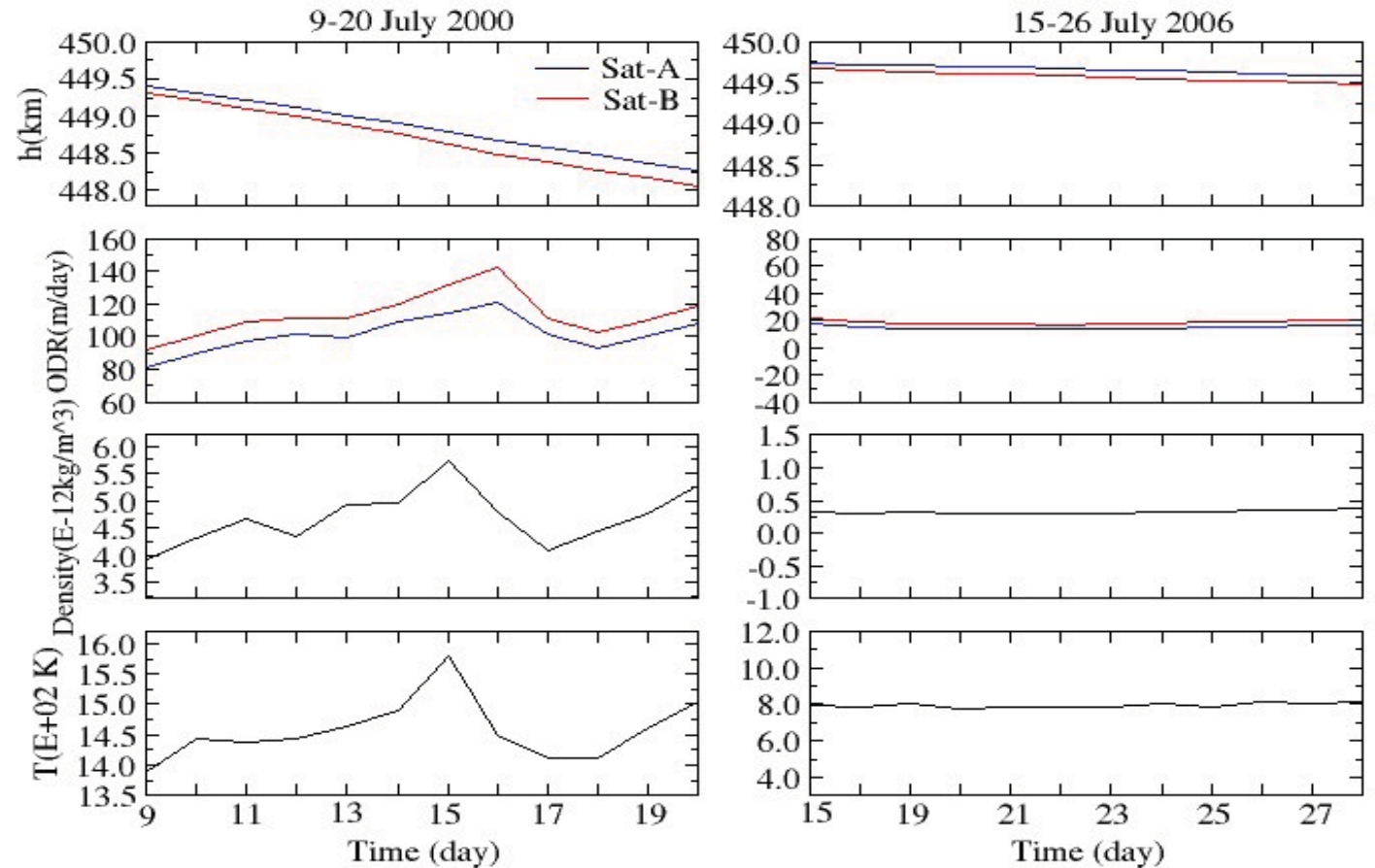

Fig. 6. Corresponding mean daily variations in h, ODR, thermospheric T and $\rho$ for Sat-A and Sat-B during (i) 9-20 July 2000 and (ii) $15-26$ July 2006

the former regime, while the respective decay for the latter regime are $0.16 \mathrm{~km}$ and $0.20 \mathrm{~km}$. The respective mean ODR are $101.38 \mathrm{~m} /$ day and $113.22 \mathrm{~m} /$ day (for elevated activity), and $14.72 \mathrm{~m} /$ day and $18.52 \mathrm{~m} /$ day (exceptionally quiet).

Sat-B has larger values of height decay $(\mathrm{h})$ and ODR in both regimes, and therefore affected by atmospheric drag force more than Sat-A, because its ballistic coefficient is higher. The results of our simulation confirm (i) the dependence of atmospheric drag force on satellites ballistic coefficient, and (ii) geomagnetic storms being the leading driver of large-scale disturbances in the coupled MIT and consequently the leading 'perturber' of satellites' motion in low Earth orbit. Our model can be useful for situational awareness and mitigation of the potential threat posed by solar activity in modulating satellites trajectories.

\section{ACKNOWLEDGMENT}

We acknowledge the use of NASA/GSFC's Space Physics Data Facility's OMNIWeb servicefor the 1-hour averaged values of $V_{s w}, P D, D s t$, IMF $B_{y}$ and $B_{z}$, and $A E$. 


\section{REFERENCES}

[1] J. Wertz, and W.J. Larson, Space Mission Analysis and Design, 3rd ed. Kluwer Academy, El Segundo, Calif, 145, 1999.

[2] V.A. Chobotov, Orbital Mechanics, 3rd ed. AIAA Education series. American Institute of Aeronautics Inc, Virginia, 193-213, 2002.

[3] V.U.J. Nwankwo, Effects of Space Weather on Earth's Ionosphere and Nominal LEO Satellites' Aerodynamic Drag, (PhD Thesis). University of Calcutta, Kolkata, India, 2016.

[4] V.U.J. Nwankwo, S.K. Chakrabarti and R.S. Weigel, "Effects of plasma drag on low Earth orbiting Satellites due to solar forcing induced perturbations and heating," Adv. Space Res. 56, 47-56, 2015.

[5] V.U.J. Nwankwo and K.S. Chakrabarti, "Effects of plasma drag on low earth orbitingsatellites due to heating of earth's atmosphere by coronal mass ejections," arXiv:1305.0233c1 [physics.space-hp], 2013.

[6] R.S. Jakhu, Iridium-Cosmos collision and its implications for space operations. In Kai-UweSchrogl, et al (eds.), Yearbook on Space Policy 2008/2009, Springer, Wien New York, 2010, pp 254-275, 2009.

[7] I. McCrea, (Science and Technology Facilities council, RAL Space, Wiltshire, UK), Letter to: Newton International Fellowship (The Royal Society, UK). 2018 Mar. 21. 1 Leaf. Located at: 6-9 Carlton House, Terrace, London SW1Y.

[8] V.U.J. Nwankwo, N.N. Jibiri and M.T. Kio, The impact of space radiation environment on satellites operation in near-Earth space. In
Jonathan Becedas (Ed), Satellites and Innovative Technology. ISBN 987-1-78985-862-4. InTechOpen Publishing London, 2019

[9] G.W. Prolss, Physics of the Earth's space environment. Springer Berlin Heidelberg, Germany, 2004.

[10] V.U.J. Nwankwo and S.K. Chakrabarti, "Theoretical modeling of drag force impact on a model international space station (ISS) during variation of solar activity," Trans. JSASS Aerosp. Technol. Jpn. 12, 47-53, 2014

[11] V.U.J. Nwankwo and S.K. Chakrabarti, "Effects of space weather on the ionosphere and LEO satellites' orbital trajectory in equatorial, low and middle latitude," Adv. Space Res., 61(7), pp. 1880-1889, 2018.

[12] V.U.J Nwankwo, "Space Weather: Responses of the Atmosphere to Solar Activity and Its Implications for LEO Satellites Aerodynamic Drag." In Mukhopadhyay B., Sasmal S. (Eds.), Exploring the Universe: From Near Space to Extra-Galactic. Springer Inter. Pub., Switzerland AG, 2018.

[13] R.L. Walterscheid, Solar cycle effects on the upper atmosphere Implications for satellite drag. J. Spacecr. Rockets, 26, 439-444, 1989.

[14] J.M. Picone, A.E. Hedin, D.P. Drob and A.C. Aikin, "NRLMSISE-00 empirical model of the atmosphere: Statistical comparisons and scientific issues," J. Geophys. Res., 107, A12, pp. 1468-1484, 2002. 